\title{
Coordinate deletion of zeroes
}

\author{
Eero Räty \\ Centre for Mathematical Sciences \\ Wilberforce Road \\ Cambridge CB3 0WB, U.K. \\ epjr2@cam.ac.uk
}

Submitted: Jan 31, 2019; Accepted: Aug 19, 2019; Published: Sep 13, 2019

(C) The author. Released under the CC BY-ND license (International 4.0).

\begin{abstract}
For a family $A \subseteq\{0, \ldots, k\}^{n}$, define the $\delta$-shadow of $A$ to be the set obtained from $A$ by removing from any of its vectors one coordinate that equals zero. Given the size of $A$, how should we choose $A$ to minimise its $\delta$-shadow? Our aim in this paper is to show that, for any $r$, the family of all sequences with at most $r$ zeros has minimal $\delta$-shadow. We actually give the exact best $A$ for every size.
\end{abstract}

Mathematics Subject Classifications: 05 D05

\section{Introduction}

The classical Kruskal-Katona theorem is concerned with the lower shadow of set systems. For $A \subseteq\{0,1\}^{n}$, define the lower shadow of $A$ to be the set of sequences obtained from any of its vectors by flipping one of its 1 -entries to 0 . The rank of a sequence $x \in\{0, \ldots, n\}^{k}$ is defined to be $|x|=\sum_{i=1}^{k} x_{i}$. Note that the lower shadow operator decreases the rank of a sequence by 1 . For given $r$, it is natural to ask how to choose a family $A \subseteq\{0,1\}^{n}$ of given size containing only vectors with rank $r$ which minimises the lower shadow. This question was answered by Kruskal [4] and Katona [3].

Define the colexicographic order on $\left\{x \in\{0,1\}^{n}:|x|=r\right\}$ by $x \leqslant$ colex $y$ if $\max (X \Delta Y)$ lies in $Y$. Here $X=\left\{i: x_{i}=1\right\}$ and $Y=\left\{i: y_{i}=1\right\}$. The Kruskal-Katona theorem states that for a set $A \subseteq\{0,1\}^{n}$ containing only sequences of rank $r$, the lower shadow is minimised when $A$ is chosen to be an initial segment of the colexicographic order.

Instead of changing the coordinates, it is also natural to define an operator which acts by deleting coordinates. For $A \subseteq\{0, \ldots, k\}^{n}$ define the coordinate deletion shadow $\Delta A$ to be the set of those sequences obtained from any of its vectors by deleting one coordinate. For example $\Delta(\{000,001,002,121\})=\{00,01,02,12,11,21\}$. 
Again it is natural to ask that which sets minimise the coordinate deletion shadow. Define the simplicial order $\leqslant_{\text {sim }}$ on $\{0,1\}^{n}$ by

$$
x \leqslant_{\operatorname{sim}} y \text { if }|x|<|y| \text { or }|x|=|y| \text { and } \min (X \Delta Y) \in X \text {. }
$$

It was proved by Danh and Daykin that for subsets of $\{0,1\}^{n}, \Delta A$ is minimised by an initial segment of the simplicial order [2]. They also conjectured a certain order as best in $\{0,1, \ldots\}^{n}$, but Leck [5] showed that this turned out to be false and in fact there is no order in general whose all initial segments have minimal coordinate deletion shadow.

Bollobás and Leader [1] pointed out that for $k \geqslant 2$ the sets $A_{t}=\{0, \ldots, t-1\}^{n} \subseteq$ $\{0, \ldots, k\}^{n}$ are extremal for $\Delta$. Indeed, suppose that $B \subseteq\{0, \ldots, k\}^{n}$ is extremal with $|B|=t^{n}$. Define $B_{[n] \backslash\{i\}}$ to be the projection of $B$ onto the hyperplane excluding the $i^{t h}$ direction. Suppose that $|\Delta B|<t^{n-1}$. Since $B_{[n] \backslash\{i\}} \subseteq \Delta B$ for all $i$, it follows that $\left|B_{[n] \backslash\{i\}}\right|<t^{n-1}$ for all $i$. Thus the Loomis-Whitney inequality [6] implies that $|B|^{n-1} \leqslant$ $\left(\prod_{i=1}^{n} B_{[n] \backslash\{i\}}\right)<t^{n(n-1)}$, which contradicts $|B|=t^{n}$. Hence $|\Delta B| \geqslant t^{n-1}$ and since $\Delta A_{t}=t^{n-1}$, it follows that each $A_{t}$ is extremal.

In addition, Bollobás and Leader made the following conjecture that certain other type of sets are also extremal.

Conjecture 1 (Bollobás, Leader [1]). For each $t \leqslant k$ and $r \leqslant n$, let $B_{r, t} \subseteq\{0, \ldots, k\}^{n}$ be the subset containing all sequences with at most $r$ zeros, and with all coordinates in $\{0, \ldots, t\}$. Then the sets $B_{r, t}$ have extremal $\Delta$-shadow in $\{0, \ldots, k\}^{n}$.

Even the case $t=k$ in the conjecture in unknown.

There is, however, a notion that comes 'between' the lower shadow and the coordinate deletion shadow. The usual lower shadow operator decreases the rank by 1 and preserves the dimension $n$, while the coordinate deletion shadow decreases the dimension by 1 but there is no control on how it changes the rank. So it is natural to consider the following operator which preserves the rank, but reduces the dimension by one.

Define the $\delta$-shadow of $A \subseteq\{0, \ldots, k\}^{n}$ to be the set of sequences in $\{0, \ldots, k\}^{n-1}$ obtained by removing one coordinate that equals 0 from any of the vectors in $A$. Denote this set by $\delta A$. Thus for example $\delta(\{00011,00101\})=\{0011,0101\}$ and $\delta(\{112,113,123\})=$ $\varnothing$. For convenience, we say that $A$ has minimal $\delta$-shadow if $|\delta B| \geqslant|\delta A|$ holds for all $B \subseteq\{0, \ldots, k\}^{n}$ with $|B|=|A|$.

How can we find sets $A$ with minimal $\delta$-shadow? If $|A| \leqslant k^{n}$ then the question is trivial, as one can take any subset of $\{1, \ldots, k\}^{n}$ of given size. In general, it is natural to choose $A$ to contain sequences with as few zeros as possible. Furthermore, it is natural to guess that for each $0 \leqslant i \leqslant n$, the sets containing all sequences with at most $i$ zeros have minimal $\delta$-shadow.

Our main result in this paper is to find an order on $\{0, \ldots, k\}^{n}$ whose initial segments have minimal $\delta$-shadow. In particular, it follows that the sets containing all sequences with at most $i$ zeros have minimal $\delta$-shadow.

In order to state the main result, we need a few definitions. For a sequence $x \in$ $\{0, \ldots, k\}^{n}$, let $R(x)=\left\{i: x_{i}=0\right\}$ and let $w(x)=|R(x)|$. Let

$$
L_{r}(n)=\left\{x \in\{0, \ldots, k\}^{n}: w(x)=r\right\} .
$$


Note that $\delta$ maps sequences in $L_{r}(n)$ to sequences in $L_{r-1}(n-1)$.

For $x \in\{0, \ldots, k\}^{n}$, define its reduced sequence to be the sequence obtained by removing all coordinates from $x$ that equal 0 . Denote the reduced sequence of $x$ by $r e(x)$. Note that for any sequence $s$ and for any $t \in \delta s$ we have $r e(s)=r e(t)$, as removing a coordinate which equals 0 does not change the reduced sequence. Hence each $L_{r}(n)$ splits into disjoint components based on the reduced sequences.

We will start by proving that inside a component one should choose sequences $x$ for which the sets $R(x)$ form an initial segment of colex. This is a straightforward consequence of the work of Danh and Daykin in [2].

Since we know that $\{0, \ldots, k\}^{n}$ splits into components based on the reduced sequences, and we know that the initial segments of the colexicographic order minimise the $\delta$-shadow inside each component, we are left with the question on how to split the sequences into different components in order to minimise the shadow.

We go on to prove that in order to minimise the shadow of a subset in $\{0, \ldots, k\}^{n}$, one should first prefer sequences in components in $L_{r}(n)$ rather than in $L_{s}(n)$ for all $r<s$, and inside $L_{r}(n)$ one should choose all sequences from one component before taking any sequences from another component. As a consequence we obtain an order whose initial segments minimises the $\delta$-shadow.

For $r \in\{0,1, \ldots, k\}$ define $R_{r}(x)=\left\{i: x_{i}=r\right\}$ and $w_{r}(x)=\left|R_{r}(x)\right|$. Note that $R=R_{0}$ and $w=w_{0}$. For all $r$ we define an order $\leqslant_{c}$ on $\{1, \ldots, k\}^{r}$ as follows. For distinct $x, y \in\{1, \ldots, k\}^{r}$ let $i$ be minimal such that $R_{i}(x) \neq R_{i}(y)$. We say that $x \leqslant_{c} y$ if and only if $\max \left(R_{i}(x) \Delta R_{i}(y)\right) \in R_{i}(y)$.

Define an order $\leqslant$ on $\{0, \ldots, k\}^{n}$ as follows. For distinct $x, y \in\{0, \ldots, k\}^{n}$ we set $x \leqslant y$ if

1. $w_{0}(x)<w_{0}(y)$

2. $w_{0}(x)=w_{0}(y), r e(x) \neq r e(y)$ and $r e(x) \leqslant c r e(y)$

3. $w_{0}(x)=w_{0}(y)$, re $(x)=r e(y)$ and $R_{0}(x) \leqslant$ colex $R_{0}(y)$

Now we are ready to state our main theorem.

Theorem 2. Let $A \subseteq\{0, \ldots, k\}^{n}$ and let $B$ be an initial segment of $\leqslant$ with $|B|=|A|$. Then $|\delta A| \geqslant|\delta B|$.

In particular, it follows that the sets of the form $L_{\leqslant r}(n)=\bigcup_{i=0}^{r} L_{i}(n)$ are extremal. Note that for fixed $r$, every component of $L_{r}(n)$ behaves in the same way. Hence for any fixed $r$, one could replace the $\leqslant_{c}$-order by any other order on $\{1, \ldots, k\}^{r}$ in the definition of the $\leqslant$-order.

The plan of the paper is as follows. In Section 2 we prove that inside a component the sets of sequences whose associated sets $R(x)$ form an initial segment of colex have minimal $\delta$-shadow. In Section 3 we prove Theorem 2. In Section 4 we generalise the $\delta$ shadow to allow deleting one coordinate in some set $\{0, \ldots, r\}$ instead of just deleting one coordinate which equals 0 . In this case we show that sets $\left\{x: \sum_{i=0}^{r} w_{i}(x) \leqslant s\right\}$, which 
are analogous to the sets $L_{\leqslant s}(n)$, are extremal for all $0 \leqslant s \leqslant n$. In this general case we do not know what happens for sets of other sizes.

We use the standard notation $[n]=\{1, \ldots, n\}$ and $[n]^{(r)}=\{A \subseteq[n]:|A|=r\}$. We write $L_{r}$ instead of $L_{r}(n)$ if the dependence on $n$ is clear. When $k=1$, we may also write $\{0,1\}_{r}^{n}$ instead of $L_{r}(n)$. This notation will be used to highlight that we are working with $\{0,1\}$-sequences.

\section{Deletion on $\{0,1\}$-sequences}

In this section we always work with subsets of $\{0,1\}^{n}$ or $\{0,1\}_{r}^{n}$. Danh and Daykin proved the following result for the coordinate deletion shadow $\Delta$ on $\{0,1\}^{n}$.

Theorem 3. (Danh, Daykin [2]). Let $A \subseteq\{0,1\}^{n}$ and let $B$ be an initial segment of the simplicial order with $|B|=|A|$. Then $|\Delta A| \geqslant|\Delta B|$.

There is a natural correspondence between the sequences $\{0,1\}^{n}$ and the power-set $\mathcal{P}(\{1, \ldots, n\})$. For our purposes it will be convenient to choose this correspondence by mapping a sequence $\left(x_{i}\right)$ to the set $R_{0}(x)=\left\{i: x_{i}=0\right\}$.

In this way we can identify a set $A \subseteq\{0,1\}_{r}^{n}$ with the set system $\mathcal{A} \subseteq[n]^{(r)}$ containing the images of the elements of $A$ under this bijection. This enables us to translate the questions on $\delta$ to the questions related to the properties of the set systems $\mathcal{A} \subseteq[n]^{(r)}$ instead. We start by proving that the subsets $A$ of $\{0,1\}_{r}^{n}$ with minimal shadow are the ones whose associated set $\mathcal{A}$ is an initial segment of colex.

Lemma 4. Let $A \subseteq\{0,1\}_{r}^{n}$, and let $B \subseteq\{0,1\}_{r}^{n}$ be chosen such that $|A|=|B|$ and $\mathcal{B}$ is an initial segment of colex. Then $|\delta A| \geqslant|\delta B|$.

Proof. Define $C_{1}=A \cup L_{>r}(n)$ and $C_{2}=B \cup L_{>r}(n)$, where $L_{>r}(n)=\bigcup_{i=r+1}^{n}\{0,1\}_{i}^{n}$. Now $C_{2}$ is isomorphic to an initial segment of the simplicial order, and the isomorphism is the map which reverses the sequences. Since this map preserves the size of $\Delta$-shadow, Theorem 3 implies that $\Delta C_{2}$ is minimal and hence

$$
\left|\Delta C_{2}\right| \leqslant\left|\Delta C_{1}\right|
$$

Note that $\Delta C_{1}=L_{>r}(n-1) \cup \delta A$ and $\Delta C_{2}=L_{>r}(n-1) \cup \delta B$. Indeed, $L_{>r}(n-1)$ is certainly a subset of both of these sets, and the only contribution to the elements not in $L_{>r}(n-1)$ comes from removing 0 from a sequence which contains exactly $n-r 1$ 's. Hence

$$
\left|\Delta C_{1}\right|=\left|L_{>r}(n-1)\right|+|\delta A|
$$

and

$$
\left|\Delta C_{2}\right|=\left|L_{>r}(n-1)\right|+|\delta B| .
$$

Combining (1), (2) and (3) gives $|\delta A| \geqslant|\delta B|$, which completes the proof. 
Lemma 4 implies that inside $\{0,1\}_{r}^{n}$ the colexicographic order minimises the size of the $\delta$-shadow. Before moving on to general $k$ from $k=1$, we find a way to relate the size of $\delta A$ to the associated family $\mathcal{A}$. For convenience, from now on we say that $A \subseteq\{0,1\}_{r}^{n}$ is an initial segment of colex if the associated set system $\mathcal{A}$ is an initial segment of colex. For $\mathcal{A} \subseteq \mathcal{P}(\{1, \ldots, n\})$ define $\mathcal{A}_{1}=\{B \in \mathcal{A}: 1 \in B\}$.

Lemma 5. Let $A \subseteq\{0,1\}_{r}^{n}$ be an initial segment of colex, and let $\mathcal{A}$ be the set system associated to $A$. Then $|\delta A|=\left|\mathcal{A}_{1}\right|$.

Proof. The proof is by induction on $|A|$; note that the case $|A|=1$ is clear. Let $B$ be an initial segment with $|B|=|A|+1$, say $B=A \cup\{x\}$ with $x=x_{1} \ldots x_{n}$. First we prove that $x_{2} \ldots x_{n}$ is the only element which could be in $\delta B \backslash \delta A$.

Indeed, suppose $t \in \delta B \backslash \delta A$ and that it is obtained by removing the $k^{t h}$ coordinate of $x$. Hence $t=x_{1} \ldots x_{k-1} x_{k+1} \ldots x_{n}$ and $x_{k}=0$. Let $i=\min \left\{j: x_{j}=1\right\}$ and set $y=0 t=$ $0 x_{1} \ldots x_{k-1} x_{k+1} \ldots x_{n}$. If $i \leqslant k$, then $y_{j}=x_{j}$ for all $j \leqslant i-1$ but $y_{k}=x_{k-1}=0 \neq 1=x_{k}$, so $y<_{\text {colex }} x$. But then $t \in \delta y \subseteq \delta A$ which contradicts $t \in \delta B \backslash \delta A$.

Hence we must have $i>k$. But in this case $x_{1}=\ldots x_{k}=0$ and therefore $t=$ $0 \ldots 0 x_{k+1} \ldots x_{n}=x_{2} \ldots x_{n}$. Hence $\delta B \backslash \delta A$ is either empty or contains only $x_{2} \ldots x_{n}$.

Note that $0 x_{2} \ldots x_{n}$ is the least element with respect to colex which has $x_{2} \ldots x_{n}$ contained in its $\delta$-shadow. Hence $x_{2} \ldots x_{n} \in \delta B \backslash \delta A$ if and only if $x=0 x_{2} \ldots x_{n}$. Thus

$$
|\delta B|=\left\{\begin{array}{cc}
|\delta A|+1 & \text { if } x_{1}=0 \\
|\delta A| & \text { if } x_{1}=1
\end{array} .\right.
$$

Also $\mathcal{B}=\mathcal{A} \cup R_{0}(x)$, and the set $R_{0}(x)$ contains 1 if and only if $x_{1}=0$. Thus

$$
\left|\mathcal{B}_{1}\right|=\left\{\begin{array}{cc}
\left|\mathcal{A}_{1}\right|+1 & \text { if } x_{1}=0 \\
\left|\mathcal{A}_{1}\right| & \text { if } x_{1}=1
\end{array}\right.
$$

and hence $|\delta B|=\left|\mathcal{B}_{1}\right|$ by induction.

\section{The main theorem}

Let $H$ be the bipartite graph with vertex set $\{0, \ldots, k\}^{n} \cup\{0, \ldots, k\}^{n-1}$ and whose edges are precisely those pairs $s, t$ with $s \in\{0, \ldots, k\}^{n}$ and $t \in \delta s$. Then for $A \subseteq\{0, \ldots, k\}^{n}, \delta A$ is just the neighbourhood of $A$ in the graph $H$. Note that both classes can be partitioned as $\{0, \ldots, k\}^{n}=\bigcup_{i=0}^{n} L_{i}(n)$ and $\{0, \ldots, k\}^{n-1}=\bigcup_{i=0}^{n-1} L_{i}(n-1)$, and by definition of $\delta$ it is clear that there are edges only between $L_{i}(n)$ and $L_{i-1}(n-1)$, with the convention $L_{-1}=\varnothing$.

Let $C$ be a connected component in $H$. Suppose $C$ is non-trivial, i.e. $\{0, \ldots, k\}^{n} \cap C \subseteq$ $L_{i}(n)$ for some $i>0$. Recall that for all $x$ and for any $y \in \delta x, x$ and $y$ have the same reduced sequences. But since $C$ is a connected component, this means that every $x \in C$ has the same reduced sequence. Conversely it is easy to check that for $i>0$ all sequences $x \in L_{i}(n) \cup L_{i-1}(n-1)$ with the same reduced sequence are in the same connected 
component. Thus we can deduce that the connected components in $H$ are given as follows.

Lemma 6. For $s \in \bigcup_{i=0}^{r}\{1, \ldots, k\}^{i}$ define $C_{s}=\left\{x \in\{0, \ldots, k\}^{n}:\right.$ re $\left.(x)=s\right\}$ and $D_{s}=$ $\left\{x \in\{0, \ldots, k\}^{n-1}: \operatorname{re}(x)=s\right\}$. Then $C_{s} \cup D_{s}$ are the connected components of $H$.

Broadly speaking, we only need to understand how to minimise $\delta$ inside a connected component, and to determine how to distribute the sequences into different connected components in order to minimise $\delta$. It turns out that inside a connected component one should choose sequences $x$ whose sets $R_{0}(x)$ form an initial segment of colex.

Lemma 7. Let $C \subseteq L_{i}(n) \cup L_{i-1}(n-1)$ be a connected component corresponding to a reduced word $x=x_{1} \ldots x_{n-i}$. Let $B \subseteq L_{i} \cap C$ and let $A \subseteq L_{i} \cap C$ be a set of sequences chosen such that $|A|=|B|$ and $\left\{R_{0}(x): x \in A\right\}$ is an initial segment of colex. Then $|\delta B| \geqslant|\delta A|$.

Proof. Note that the behaviour of a connected component depends only on $n-i$ and, in particular, not on the sequence $x_{1} \ldots x_{n-i}$, as the reduced sequence and the order of coordinates in the reduced sequence are preserved under taking the $\delta$-shadow. In particular, all such connected components have the same size and they all behave in the same way under taking the $\delta$-shadow. Hence it suffices to consider only the component with $x_{1}=\cdots=x_{n-i}=1$. But this component is just $\{0,1\}_{i}^{n}$ and hence the result follows from Lemma 4.

Hence it remains to understand how to fill different connected components. Our aim is to show that it is optimal to first choose all sequences in a component before taking sequences from another component, and also to prefer a component in $L_{i}(n)$ over a component in $L_{i+1}(n)$.

From now on we call the sets $C_{s}$ connected components, i.e. by a connected component we refer to the intersection of a connected component with $\{0, \ldots, k\}^{n}$.

For $s, t \in \bigcup_{i=0}^{r}\{1, \ldots, k\}^{i}$ define the $s, t$-compression operator as follows. For $A \subseteq$ $\{0, \ldots, k\}^{n}$ its compression $B=C_{s, t}(A)$ is given by setting

1. $B \cap C_{s}$ to be an initial segment of colex of length $\min \left(\left|A \cap\left(C_{s} \cup C_{t}\right)\right|,\left|C_{s}\right|\right)$

2. $B \cap C_{t}$ to be an initial segment of colex of length $\max \left(0,\left|A \cap\left(C_{s} \cup C_{t}\right)\right|-\left|C_{s}\right|\right)$

3. $B \backslash\left(C_{s} \cup C_{t}\right)=A \backslash\left(C_{s} \cup C_{t}\right)$

It is clear that $\left|C_{s . t}(A)\right|=|A|$ for all $s$ and $t$. As usual, we say that $A \subseteq\{0, \ldots, k\}^{n}$ is $s, t$-compressed if $C_{s, t}(A)=A$.

In order to prove Theorem 2, we need the following two Lemmas.

Lemma 8. Let $A \subseteq\{0, \ldots, k\}^{n}$ be a set and let $s, t \in\{1, \ldots, k\}^{n-i}$ for some $i$. Then $|\delta A| \geqslant\left|\delta C_{s, t}(A)\right|$.

Lemma 9. Let $A \subseteq\{0, \ldots, k\}^{n}$ be a set and let $s \in\{1, \ldots, k\}^{n-i}, t \in\{1, \ldots, k\}^{n-i-1}$ for some $i$. Then $|\delta A| \geqslant\left|\delta C_{s, t}(A)\right|$. 
In order to prove these Lemmas, we relate them to the appropriate questions on the subsets of $[n]^{(i)}$. We now state these results, but the proof is presented after the proofs of Lemma 8 and Lemma 9.

Define $\mathcal{B} \subseteq[n]^{(r)}$ to be a segment if there exist initial segments $\mathcal{I}$ and $\mathcal{J}$ of colex such that $\mathcal{A}=\mathcal{I} \backslash \mathcal{J}$.

Lemma 10. The following claims are true.

Claim 1. Let $\mathcal{A} \subseteq[n]^{(i)}$ be a segment and $\mathcal{I} \subseteq[n]^{(i)}$ be an initial segment of colex with $|\mathcal{A}|=|\mathcal{I}|$. Then $\left|\mathcal{A}_{1}\right| \leqslant\left|\mathcal{I}_{1}\right|$

Claim 2. Let $\mathcal{I} \subseteq[n]^{(i)}$ and $\mathcal{J} \subseteq[n]^{(i+1)}$ be initial segments of colex with $|\mathcal{I}|=|\mathcal{J}|$. Then $\left|\mathcal{I}_{1}\right| \leqslant\left|\mathcal{J}_{1}\right|$

Claim 3. Let $\mathcal{A} \subseteq[n]^{(r)}$ be a segment and let $\mathcal{I}=[n]^{(r)} \backslash \mathcal{J}$, where $\mathcal{J}$ is an initial segment of colex chosen such that $|\mathcal{I}|=|\mathcal{A}|$. Then $\left|\mathcal{A}_{1}\right| \geqslant\left|\mathcal{I}_{1}\right|$.

Claim 4. Let $\mathcal{I}_{*}$ and $\mathcal{J}_{*}$ be initial segments of colex chosen such that $\mathcal{I}=[n]^{(i)} \backslash \mathcal{I}_{*}$ and $\mathcal{J}=[n]^{(i+1)} \backslash \mathcal{J}_{*}$ satisfies $|\mathcal{I}|=|\mathcal{J}|$. Then $\left|\mathcal{I}_{1}\right| \leqslant\left|\mathcal{J}_{1}\right|$.

Proof of Lemma 8. Let $A \subseteq\{0, \ldots, k\}^{n}$ and $B=C_{s, t}(A)$. Note that $B$ depends only on $\left|A \cap C_{s}\right|$ and $\left|A \cap C_{t}\right|$. Lemma 7 implies that an initial segment of colex minimises the $\delta$-shadow inside a connected component, so we may assume that $Q=A \cap C_{s}$ and $R=A \cap C_{t}$ are initial segments of colex.

Let $S=B \cap C_{s}$ and $T=B \cap C_{t}$. Let $\mathcal{Q}, \mathcal{R}, \mathcal{S}$ and $\mathcal{T}$ be the associated families in $[n]^{(i)}$. Since $B \backslash\left(C_{s} \cup C_{t}\right)=A \backslash\left(C_{s} \cup C_{t}\right)$, it follows that $|\delta A| \geqslant|\delta B|$ is equivalent to $|\delta Q|+|\delta R| \geqslant|\delta S|+|\delta T|$. By applying Lemma 5 , this can be rewritten as $\left|\mathcal{Q}_{1}\right|+\left|\mathcal{R}_{1}\right| \geqslant\left|\mathcal{S}_{1}\right|+\left|\mathcal{T}_{1}\right|$.

Case 1: $|Q|+|R| \leqslant\left|C_{s}\right|$.

By definition of $B$, it follows that $T=\varnothing$ and $|S|=|Q|+|R|$. Let $\mathcal{I}=\mathcal{S} \backslash \mathcal{Q}$. Since $\mathcal{S}$ and $\mathcal{Q}$ are initial segments of colex, it follows that $\mathcal{I}$ is a segment of length $|\mathcal{R}|$. Thus $\left|\mathcal{I}_{1}\right| \leqslant\left|\mathcal{R}_{1}\right|$ by Claim 1 and hence

$$
\left|\mathcal{S}_{1}\right|+\left|\mathcal{T}_{1}\right|=\left|\mathcal{Q}_{1}\right|+\left|\mathcal{I}_{1}\right| \leqslant\left|\mathcal{Q}_{1}\right|+\left|\mathcal{R}_{1}\right|
$$

as required.

Case 2: $|Q|+|R|>\left|C_{s}\right|$.

In this case $S=C_{s}$ and hence $|T|<|R|$. Thus we can write $\mathcal{I}=\mathcal{R} \backslash \mathcal{T}$, which is a segment as $\mathcal{R}$ and $\mathcal{T}$ are initial segments of colex. Also set $\mathcal{J}=\mathcal{S} \backslash \mathcal{Q}=[n]^{(i)} \backslash \mathcal{Q}$, which is a segment as well. Since $|\mathcal{S}|+|\mathcal{T}|=|\mathcal{R}|+|\mathcal{Q}|$ it follows that $|\mathcal{I}|=|\mathcal{J}|$. Thus Claim 3 implies that $\left|\mathcal{J}_{1}\right| \leqslant\left|\mathcal{I}_{1}\right|$. 
Combining this together with the definitions of $\mathcal{I}$ and $\mathcal{J}$ implies that

$$
\left|\mathcal{Q}_{1}\right|+\left|\mathcal{R}_{1}\right|=\left|\mathcal{Q}_{1}\right|+\left|\mathcal{I}_{1}\right|+\left|\mathcal{T}_{1}\right| \leqslant\left|\mathcal{Q}_{1}\right|+\left|\mathcal{J}_{1}\right|+\left|\mathcal{T}_{1}\right|=\left|\mathcal{S}_{1}\right|+\left|\mathcal{T}_{1}\right|
$$

which completes the proof.

Proof of Lemma 9. Let $A \subseteq\{0, \ldots, k\}^{n}$ and $B=C_{s, t}(A)$. By Lemma 7 we may assume that both $A \cap C_{s}$ and $A \cap C_{t}$ are initial segments of colex. As in the proof of Lemma 8, set $Q=A \cap C_{s}, R=A \cap C_{t}, S=B \cap C_{s}$ and $T=B \cap C_{t}$. Let $\mathcal{Q}$ and $\mathcal{S}$ be the associated set systems in $[n]^{(i)}$, and $\mathcal{R}$ and $\mathcal{T}$ be the associated set systems in $[n]^{(i+1)}$. By Lemma 5 it suffices to prove that $\left|\mathcal{Q}_{1}\right|+\left|\mathcal{R}_{1}\right| \geqslant\left|\mathcal{S}_{1}\right|+\left|\mathcal{T}_{1}\right|$.

Case 1: $|\mathcal{Q}|+|\mathcal{R}| \leqslant\left|C_{s}\right|$.

By definition of $B$, it follows that $\mathcal{S}$ is an initial segment of colex of length $|\mathcal{Q}|+|\mathcal{R}|$ in $[n]^{(i)}$, and $\mathcal{T}=\varnothing$. Let $\mathcal{I}$ be an initial segment of colex of length $|\mathcal{R}|$ in $[n]^{(i)}$, and set $\mathcal{J}=\mathcal{S} \backslash \mathcal{Q}$. Then $|\mathcal{J}|=|\mathcal{R}|=|\mathcal{I}|$ and $\mathcal{J}$ is a segment, as $\mathcal{S}$ and $\mathcal{Q}$ are initial segments of colex. Thus Claim 1 implies that $\left|\mathcal{J}_{1}\right| \leqslant\left|\mathcal{I}_{1}\right|$. On the other hand, Claim 2 implies that $\left|\mathcal{R}_{1}\right| \geqslant\left|\mathcal{I}_{1}\right|$. Combining these two gives $\left|\mathcal{R}_{1}\right| \geqslant\left|\mathcal{J}_{1}\right|$. Hence

$$
\left|\mathcal{S}_{1}\right|+\left|\mathcal{T}_{1}\right|=\left|\mathcal{S}_{1}\right|=\left|\mathcal{J}_{1}\right|+\left|\mathcal{Q}_{1}\right| \leqslant\left|\mathcal{R}_{1}\right|+\left|Q_{1}\right|,
$$

as required.

Case 2: $|\mathcal{Q}|+|\mathcal{R}|>\left|C_{s}\right|$.

By definition of $B$ it follows that $\mathcal{S}=[n]^{(i)}$. Note that since $|\mathcal{S}| \geqslant|\mathcal{Q}|$, it follows that $|\mathcal{R}| \geqslant|\mathcal{T}|$. Hence $\mathcal{I}=\mathcal{R} \backslash \mathcal{T} \subseteq[n]^{(i+1)}$ is a segment and it satisfies $\mathcal{R}=\mathcal{I} \cup \mathcal{T}$. Let $\mathcal{I}_{*} \subseteq[n]^{(i+1)}$ be an initial segment of colex chosen such that $\mathcal{K}=[n]^{(i+1)} \backslash \mathcal{I}_{*}$ is a segment of size $|\mathcal{I}|$. Define $\mathcal{J}=[n]^{(i)} \backslash \mathcal{Q}=\mathcal{S} \backslash \mathcal{Q}$. Hence $\mathcal{J}$ is a segment of size $|\mathcal{S}|-|\mathcal{Q}|=\left|\mathcal{R}_{1}\right|-\left|\mathcal{T}_{1}\right|=|\mathcal{I}|$

Claim 3 implies that $\left|\mathcal{I}_{1}\right| \geqslant\left|\mathcal{K}_{1}\right|$ and Claim 4 implies that $\left|\mathcal{K}_{1}\right| \geqslant\left|\mathcal{J}_{1}\right|$. Thus combining these results gives $\left|\mathcal{I}_{1}\right| \geqslant\left|\mathcal{J}_{1}\right|$. Using the definitions of $\mathcal{I}$ and $\mathcal{J}$ it follows that

$$
\left|\mathcal{S}_{1}\right|+\left|\mathcal{T}_{1}\right|=\left|\mathcal{J}_{1}\right|+\left|\mathcal{Q}_{1}\right|+\left|\mathcal{T}_{1}\right| \leqslant\left|\mathcal{I}_{1}\right|+\left|\mathcal{Q}_{1}\right|+\left|\mathcal{T}_{1}\right|=\left|\mathcal{R}_{1}\right|+\left|\mathcal{Q}_{1}\right|
$$

which completes the proof of Lemma 9.

Proof of Lemma 10. We start by proving Claim 1, and then we will prove that other claims can be deduced from Claim 1.

Proof of Claim 1. Since $\mathcal{A}$ is a segment, there exist initial segments $\mathcal{I}_{A}$ and $\mathcal{J}_{A}$ of colex with $\mathcal{A}=\mathcal{I}_{A} \backslash \mathcal{J}_{A}$, and denote their associated sets of sequences by $I_{A}$ and $J_{A}$. Let $C$ be obtained from $J_{A}$ by reversing all the sequences and by adding $2 n 1$ 's at the start of 
each reversed sequence. Let $D$ be obtained from $I$ by adding $2 n 1$ 's at the end of each sequence in $I$, where $I$ is the set of sequences associated to $\mathcal{I}$. Set $B=C \cup D$.

Due to the additional 1's at the start of the elements of $C$ and at the end of the elements of $D$, it follows that $\delta C$ and $\delta D$ are disjoint sets. Also note that reversing all the sequences and adding 1 's to every sequence do not change the size of the shadow. Hence $|\delta B|=|\delta C|+|\delta D|=|\delta I|+\left|\delta J_{A}\right|$. On the other hand, since $\mathcal{I}$ and $\mathcal{J}_{A}$ are initial segments of colex, Lemma 5 implies that $|\delta I|=\left|\mathcal{I}_{1}\right|$ and $\left|\delta J_{A}\right|=\left|\left(\mathcal{J}_{A}\right)_{1}\right|$. Thus

$$
|\delta B|=\left|\mathcal{I}_{1}\right|+\left|\left(\mathcal{J}_{A}\right)_{1}\right| .
$$

Since $\mathcal{I}_{A}$ is an initial segment of colex, Lemma 5 implies that $\left|\delta I_{A}\right|=\left|\left(\mathcal{I}_{A}\right)_{1}\right|$. But $\mathcal{I}_{A}$ is a disjoint union of $\mathcal{J}_{A}$ and $\mathcal{A}$ so

$$
\left|\delta I_{A}\right|=\left|\left(\mathcal{I}_{A}\right)_{1}\right|=\left|\left(\mathcal{J}_{A}\right)_{1}\right|+\left|\mathcal{A}_{1}\right| \text {. }
$$

Since $\mathcal{I}_{A}$ is an initial segment of colex, the corresponding set of sequences $I_{A}$ has minimal shadow inside a connected component. Since $|\mathcal{B}|=\left|\mathcal{I}_{A}\right|$, it follows that

$$
|\delta B| \geqslant\left|\delta I_{A}\right| \text {. }
$$

Thus combining (4), (5) and (6) gives

$$
\left|\mathcal{I}_{1}\right| \geqslant\left|\mathcal{A}_{1}\right|
$$

as required.

Claim $1 \Rightarrow$ Claim 3. Let $\mathcal{A}$ and $\mathcal{I}$ be as in Claim 3. Define $\overline{\mathcal{A}}=\left\{A^{c}: A \in \mathcal{A}\right\}$ and define $\overline{\mathcal{I}}$ similarly. Note that $|\overline{\mathcal{A}}|=|\mathcal{A}|$ and $\overline{\mathcal{A}} \subseteq[n]^{(n-r)}$. It is easy to check that if $\mathcal{B} \subseteq[n]^{(r)}$ is an initial segment of colex, then so is $\overline{\left([n]^{(r)} \backslash \mathcal{B}\right)}$. Thus $\overline{\mathcal{I}}$ is an initial segment of colex.

Since $\mathcal{A}$ is a segment, there exist initial segments $\mathcal{K}$ and $\mathcal{L}$ such that $\mathcal{A}=\mathcal{K} \backslash \mathcal{L}$. This can be rewritten as $\mathcal{A}=\left([n]^{(r)} \backslash \mathcal{L}\right) \backslash\left([n]^{(r)} \backslash \mathcal{K}\right)$ and hence

$$
\overline{\mathcal{A}}=\overline{\left([n]^{(r)} \backslash \mathcal{L}\right) \backslash\left([n]^{(r)} \backslash \mathcal{K}\right)}=\overline{\left([n]^{(r)} \backslash \mathcal{L}\right)} \backslash \overline{\left([n]^{(r)} \backslash \mathcal{K}\right)} .
$$

As $\overline{\left([n]^{(r)} \backslash \mathcal{L}\right)}$ and $\overline{\left([n]^{(r)} \backslash \mathcal{K}\right)}$ are initial segments of colex, it follows that $\overline{\mathcal{A}}$ is a segment as well. Hence $\overline{\mathcal{A}}$ and $\overline{\mathcal{I}}$ satisfy the conditions of Claim 1 , and therefore

$$
\left|(\overline{\mathcal{I}})_{1}\right| \geqslant\left|(\overline{\mathcal{A}})_{1}\right|
$$

Note that for any set system $\mathcal{B}$ we have $|\mathcal{B}|=\left|\mathcal{B}_{1}\right|+\left|(\overline{\mathcal{B}})_{1}\right|$, as for every $A \in \mathcal{B}$ exactly one of $1 \in A$ and $1 \in A^{c}$ is satisfied. Thus

$$
|\mathcal{I}|=\left|\mathcal{I}_{1}\right|+\left|(\overline{\mathcal{I}})_{1}\right|
$$


and

$$
|\mathcal{A}|=\left|\mathcal{A}_{1}\right|+\left|(\overline{\mathcal{A}})_{1}\right|
$$

Combining (8), (9) and (10) with $|\mathcal{I}|=|\mathcal{A}|$ gives

$$
\left|\mathcal{A}_{1}\right| \geqslant\left|\mathcal{I}_{1}\right|
$$

which completes the proof of Claim 3.

Claim $1 \Rightarrow$ Claim 2. Let $\mathcal{I}$ and $\mathcal{J}$ be as in Claim 2. For $i+1 \leqslant j \leqslant n$ let $\mathcal{S}_{j}=$ $\{A \backslash\{j\}: A \in \mathcal{J}, \max A=j\}$. Thus $\mathcal{S}_{j} \subseteq[j-1]^{(i)} \subseteq[n]^{(i)}$ for all $i$. Since $\mathcal{J}$ is an initial segment of colex, it follows that $\mathcal{S}_{j}$ is an initial segment of colex in $[j-1]^{(i)}$ for all $j$. $\mathcal{S}_{j}$ is an initial segment of colex also in $[n]^{(i)}$ as initial segments of colex are not affected by adding new larger elements to the ground set. Note that we can express $\mathcal{J}$ as a disjoint union $\mathcal{J}=\bigcup_{j=i+1}^{n}\left(\mathcal{S}_{j}+\{j\}\right)$. Hence

$$
\left|\mathcal{J}_{1}\right|=\sum_{j=i+1}^{n}\left|\left(\mathcal{S}_{j}+\{j\}\right)_{1}\right|=\sum_{j=i+1}^{n}\left|\left(\mathcal{S}_{j}\right)_{1}\right| .
$$

Since each $\mathcal{S}_{j}$ is an initial segment of colex in $[n]^{(i)}$ and we have $\sum_{j=i+1}^{n}\left|\mathcal{S}_{j}\right|=|\mathcal{J}|=|\mathcal{I}|$, a repeated application of Claim 1 implies that $\left|\mathcal{J}_{1}\right| \geqslant\left|\mathcal{I}_{1}\right|$.

Claim $2 \Rightarrow$ Claim 4. Let $\mathcal{I}, \mathcal{J}, \mathcal{I}_{*}$ and $\mathcal{J}_{*}$ be as in the statement of Claim 4. Since $\mathcal{I}_{*}$ and $\mathcal{J}_{*}$ are initial segments of colex, the observation pointed out in the proof of Claim 1 $\Rightarrow$ Claim 3 implies that $\overline{\mathcal{I}} \subseteq[n]^{(n-i)}$ and $\overline{\mathcal{J}} \subseteq[n]^{(n-i-1)}$ are initial segments of colex as well. Thus Claim 2 implies that

$$
\left|(\overline{\mathcal{I}})_{1}\right| \geqslant\left|(\overline{\mathcal{J}})_{1}\right|
$$

Combining this with

$$
|\mathcal{I}|=\left|\mathcal{I}_{1}\right|+\left|(\overline{\mathcal{I}})_{1}\right|
$$

and

$$
|\mathcal{J}|=\left|\mathcal{J}_{1}\right|+\left|(\overline{\mathcal{J}})_{1}\right|
$$

gives $\left|\mathcal{J}_{1}\right| \geqslant\left|\mathcal{I}_{1}\right|$, as required.

This completes the proof of Lemma 10.

We are now ready to deduce Theorem 2. For convenience, we recall the definition of the order $\leqslant$ and restate Theorem 2 . For distinct $x, y \in\{0, \ldots, k\}^{n}$ we set $x \leqslant y$ if

1. $w_{0}(x)<w_{0}(y)$

2. $w_{0}(x)=w_{0}(y)$, re $(x) \neq r e(y)$ and $r e(x) \leqslant_{c} r e(y)$

3. $w_{0}(x)=w_{0}(y)$, re $(x)=\operatorname{re}(y)$ and $R_{0}(x) \leqslant_{\text {colex }} R_{0}(y)$ 
Theorem 2. Let $A \subseteq\{0, \ldots, k\}^{n}$ and let $B$ be an initial segment of $\leqslant$ with $|B|=|A|$. Then $|\delta A| \geqslant|\delta B|$.

Proof of Theorem 2. Let $A$ be a subset of $\{0, \ldots, k\}^{n}$ of given size with minimal $\delta A$. Define

$$
v(A)=\sum_{j=0}^{n} j\left|A \cap L_{j}(n)\right| .
$$

If possible, choose $l \in[n], s \in\{1, \ldots, k\}^{n-l}$ and $t \in\{1, \ldots, k\}^{n-l-1}$ for which $C_{s, t}(A) \neq A$. Then by Lemma $9, B=C_{s, t}(A)$ satisfies $|\delta A| \geqslant|\delta B|$ and by minimality of $\delta A$ it follows that $\delta B$ is also minimal. We also have $v(A)>v(B)$, which follows from the definition of $C_{s, t}(A)$ and from the fact that $C_{s, t}(A) \neq A$.

Repeating this process we obtain a set $A_{1}$ of size $|A|$ with minimal $\delta A_{1}$ for which $C_{s, t}\left(A_{1}\right)=A_{1}$ for all $i, s \in\{1, \ldots, k\}^{n-l}$ and $t \in\{1, \ldots, k\}^{n-l-1}$. This follows from the fact that $v(B)$ is always a non-negative integer which strictly decreases on each step. Since $C_{s, t}\left(A_{1}\right)=A_{1}$ for all $l \in[n], s \in\{1, \ldots, k\}^{n-l}$ and $t \in\{1, \ldots, k\}^{n-l-1}$, it is easy to check that there exists $i$ such that $L_{j}(n) \subseteq A_{1}$ for all $j<i$ and $L_{j}(n) \cap A_{1}=\varnothing$ for all $j>i$.

Let $C_{s_{1}}, \ldots, C_{s_{t}}$ be the connected components in $L_{i}(n)$ with $s_{j} \leqslant_{c} s_{k}$ for $j \leqslant k$. Define

$$
w(B)=\sum_{j=1}^{t} j\left|C_{s_{j}} \cap B\right| .
$$

If possible, choose $j<k$ for which $C_{s_{j}, s_{k}}\left(A_{1}\right) \neq A_{1}$, and set $B=C_{s_{j}, s_{k}}\left(A_{1}\right)$. Now $\left|\delta A_{1}\right| \geqslant|\delta B|$ by Lemma 8 and hence $\delta B$ is also minimal. Also $w\left(A_{1}\right)>w(B)$ follows directly from the definition of the compression operator and from the definition of $B$. Repeating this process we obtain a set $A_{2}$ for which

1. $\delta A_{2}$ is minimal

2. There exists $i$ such that $L_{j}(n) \subseteq A_{2}$ for all $j<i$ and $L_{j}(n) \cap A_{2}=\varnothing$ for all $j>i$

3. $C_{s_{j}, s_{k}}\left(A_{2}\right)=A_{2}$ for all $j<k$

Note that the process must terminate as $w(B)$ is always a non-negative integer which strictly decreases on each step. Since $C_{s_{j}, s_{k}}\left(A_{2}\right)=A_{2}$ for all $j<k$, it follows that there exists $p$ for which $C_{s_{k}} \subseteq A_{2}$ for all $k<p$ and $C_{s_{k}} \cap A_{2}=\varnothing$ for all $k>p$.

Let $D=A_{2} \cap C_{s_{p}}$ and let $A_{3}$ be the set obtained from $A_{2}$ by taking $A_{3} \cap C_{s_{p}}$ to be the set corresponding to an initial segment of colex of length $|D|$, and taking $A_{3} \backslash C_{s_{p}}=A_{2} \backslash C_{s_{p}}$. Then Lemma 4 implies that $\left|\delta A_{2}\right| \geqslant\left|\delta A_{3}\right|$, so $\delta A_{3}$ is minimal. On the other hand, by the construction of $A_{3}$ it is clear that $A_{3}$ is an initial segment of $\leqslant$. Hence an initial segment of $\leqslant$ minimises $\delta$. 


\section{An extremal result for the generalised shadow}

So far we have considered the operator which allows us to delete a coordinate which equals 0 . It is natural to ask what happens if we generalise this set-up and allow the deletion of any coordinate that is in some chosen set.

Define the $\delta_{r}$-shadow of $A \subseteq\{0, \ldots, k\}^{n}$ to be the subset of sequences in $\{0, \ldots, k\}^{n-1}$ obtained from any of its vectors by removing exactly one coordinate that is one of $\{0, \ldots, r\}$. Thus $\delta=\delta_{0}$ and $\Delta=\delta_{k}$. Define $v_{r}(x)=\sum_{i=0}^{r} w_{i}(x)$. That is, $v_{r}(x)$ is the number of coordinates of $x$ in the set $\{0, \ldots, r\}$. Define

$$
L_{s}(n)=\left\{x \in\{0, \ldots, k\}^{n}: v_{r}(x)=s\right\}
$$

and $L_{\leqslant s}(n)=\bigcup_{i=0}^{s} L_{i}(n)$. The aim of this section is to prove that the sets $L_{\leqslant s}(n)$ are extremal for $\delta_{r}$. This follows directly from the following Proposition.

Proposition 11. Let $A \subseteq\{0, \ldots, k\}^{n}$ and let $A_{s}=A \cap L_{s}(n)$. Then

$$
|\delta A| \geqslant \frac{1}{n(r+1)} \sum_{s=0}^{n} s\left|A_{s}\right| .
$$

Proof. Let $X=\{0, \ldots, k\}^{n}, Y=\{0, \ldots, k\}^{n-1}$, let $H$ be defined as in Section 3 and let $\mathcal{H}$ be a bipartite multigraph on $X \cup Y$ with edges given as follows. For each $x \in X \cap L_{s}(n)$ there are exactly $s$ coordinates $x_{i_{1}}, \ldots, x_{i_{s}}$ which are elements of $\{0, \ldots, r\}$. Define $y_{j}$ to be the sequence obtained by deleting the coordinate $x_{i_{j}}$. Then we certainly have $y_{j} \in \delta x$, and some of the $y_{j}$ may be equal. Define the edges of $\mathcal{H}$ to be the edges $x y_{j}$ for all $1 \leqslant j \leqslant s$ counting with multiplicities. For example, when $r=1$ the sequence $x=00121$ is connected by two edges to 0121, and by one edge to both 0012 and 0021 .

It is easy to verify that for all $y \in Y, y$ has degree $n(r+1)$ as this corresponds to adding any element of $\{0, \ldots, r\}$ to any of the $n$ possible places in the sequence $y$. Denote the neighbourhood of $x$ in $\mathcal{H}$ by $N_{\mathcal{H}}(x)$. Note that for all $x \in X$ we have $N_{\mathcal{H}}(x)=\delta x$, and hence for any $A \subseteq X$ we have $\delta A=N_{\mathcal{H}}(A)$. By the definition of $\mathcal{H}$ we have $d(x)=s$ for all $x \in L_{s}(n)$, and as observed earlier we have $d(y)=n(r+1)$ for all $y \in Y$. Since the connected components of $\mathcal{H}$ are contained in the sets $L_{s}(n) \cup L_{s-1}(n-1)$, we have $N_{\mathcal{H}}(A) \cap L_{s-1}(n-1)=N_{\mathcal{H}}\left(A \cap L_{s}(n)\right)$ and therefore

$$
\left|N_{\mathcal{H}} A\right|=\sum_{s=0}^{r}\left|N_{\mathcal{H}}\left(A_{s}\right)\right| .
$$

For a set $B \subseteq L_{s}(n)$ we have

$$
s|B|=e\left(B, N_{\mathcal{H}}(B)\right) \leqslant e\left(N_{\mathcal{H}}(B), X\right)=\left|N_{\mathcal{H}}(B)\right| n(r+1)
$$

and hence

$$
\left|N_{\mathcal{H}}(B)\right| \geqslant \frac{s}{n(r+1)}|B|
$$


Applying (17) to each term of the sum in (16) gives

$$
|\delta A|=\left|N_{\mathcal{H}} A\right| \geqslant \frac{1}{n(r+1)} \sum_{s=0}^{r} s\left|A_{s}\right|,
$$

which completes the proof.

Now we are ready to conclude that the sets $L_{\leqslant s}(n)$ are extremal.

Corollary 12. If $A \subseteq\{0, \ldots, k\}^{n}$ and $|A|=\left|L_{\leqslant s}(n)\right|$, then $|\delta A| \geqslant\left|\delta L_{\leqslant s}(n)\right|$ with equality if and only if $A=L_{\leqslant s}(n)$.

Proof. Let $B=L_{\leqslant s}(n)$. We first check that the equality holds for $B$ in (18). Note that $B_{i}=L_{i}(n)$ for all $i \leqslant s$ and $B_{i}=\varnothing$ for all $i>s$. For $i \leqslant s,\left|B_{i}\right|=\left|L_{i}(n)\right|=$ $\left(\begin{array}{c}n \\ i\end{array}\right)(r+1)^{i}(k-r)^{n-i}$ and $\left|\delta B_{i}\right|=\left|L_{i-1}(n-1)\right|=\left(\begin{array}{c}n-1 \\ i-1\end{array}\right)(r+1)^{i-1}(k-r)^{n-i}$. Therefore $\left|\delta B_{i}\right|=\frac{i}{n(r+1)}\left|B_{i}\right|$ holds for all $i \leqslant s$, and in fact holds also for $i>s$ as in this case both sides are 0 . Hence the equality holds in (17) for all $i$, and thus the equality holds in (18) as well.

Given a set $A$ of fixed size with $\left|A_{i}\right| \leqslant\left|L_{i}(n)\right|$ for all $i$, it is easy to see that $\frac{1}{n(r+1)} \sum_{t=0}^{r} t\left|A_{t}\right|$ is minimised if and only if $A=L_{\leqslant n} \cup B$ for suitably chosen $n$ and for any $B \subseteq L_{n+1}$ of suitable size. Hence given $A$ with $|A|=\left|L_{\leqslant s}(n)\right|$, the quantity $\frac{1}{n(r+1)} \sum_{t=0}^{r} t\left|A_{t}\right|$ attains its minimum value uniquely when $A=L_{\leqslant s}(n)$.

Thus

$$
|\delta A| \geqslant \frac{1}{n(r+1)} \sum_{t=0}^{r} t\left|A_{t}\right| \geqslant \frac{1}{n(r+1)} \sum_{t=0}^{s} t\left|L_{t}(n)\right|=\left|\delta L_{\leqslant s}(n)\right|,
$$

and the second inequality holds if and only if $A=L_{\leqslant s}(n)$, which completes the proof.

\section{References}

[1] B. Bollobás, I. Leader. Personal communication, 2017.

[2] T. Danh, D. Daykin. Ordering integer vectors for coordinate deletion. Journal of the London Mathematical Society, 55(3):417-426, 1997.

[3] G. Katona. A theorem of finite sets. Theory of Graphs, Akademiai Kiado, Budapest, 187-207, 1968.

[4] J. Kruskal. The number of simplices in a complex. Mathematical Optimization Techniques, University of California Press, 251-278, 1963.

[5] U. Leck. Nonexistence of a Kruskal-Katona type theorem for subword orders. Combinatorica, 24: 305-312, 2004.

[6] L. Loomis, H. Whitney. An inequality related to the isoperimetric inequality. Bulletin of the American Mathematical Society, 55:961-962, 1949. 